\title{
UN DESAFÍO PARA MÉXICO: LA CULTURA DE LA PAZ*
}

A CHALLENGE FOR MEXICO: THE
CULTURE OF PEACE

UM DESAFIO PARA O MÉXICO: A CULTURA DA PAZ
Esperanza Loera Ochoa ${ }^{a}$ esperloera@hotmail.com Thais Loera Ochoab thaisloera1@hotmail.com

Fecha de recepción: 20 de septiembre de 2016

Fecha de revisión: 20 de octubre de 2016 Fecha de aceptación: 28 de febrero de 2017

\section{RESUMEN}

El artículo realiza un estudio del control administrativo realizado a El presente trabajo tiene como finalidad desarrollar el tema de la Cultura de la Paz, como un factor indispensable y base del éxito del nuevo paradigma constitucional de justicia en México, que se dio con motivo de las reformas constitucionales en materia de derechos humanos del 11 de junio del 2011, las cuales impactan al ordenamiento jurídico mexicano, tanto teórica como normativamente. Así, el tema de los derechos humanos, se convierte no solo en modelo jurídico, sino en una forma de vida. En la primera parte del trabajo se expone un marco teórico del tema, para posteriormente establecer la internacionalización de la cultura de la paz y su relación directa con la educación, concluyendo con las aproximaciones existentes entre el tema de la paz y los derechos humanos. Se desarrolla también la educación en materia de derechos humanos y su proceso educativo, estableciendo al final del trabajo las conclusiones personales de los temas tratados.

*Artículo de reflexión.

a. Dra. en derecho profesora tiempo completo de la Division de estudios jurídicos de la Universidad de Guadalajara.

b. Dra. en derecho profesora tiempo completo de la Division de estudios jurídicos de la Universidad de Guadalajara.
MISIÓN JURÍDICA

Revista de Derecho y Ciencias Sociales

Bogotá, D.C. (Colombia)

Colaboradores Externos Internacionales

Núm. 13 Año 2017

Julio - Diciembre, pp. 189 - 208

ISSN 1794-600X 


\section{PALABRAS CLAVE}

Justicia, cultura, derechos humanos, paz, educación.

\begin{abstract}
This paper aims to elaborate on the subject of how the Culture of Peace, as an indispensable factor and base for the success of this model of justice that the new paradigm of justice in Mexico requires, should become a model to follow. In the first part of the work a theoretical framework on the subject is exposed to later establish the internationalization of the Culture of Peace and its direct relationship with education, concluding with existing approaches between the theme of peace and human rights, developing aspects that relate to education on human rights and its educational process, establishing at the end of this work the personal conclusions of the topics covered.
\end{abstract}

\section{KEY WORDS}

Justice, culture, human rights, peace, education .

\section{RESUMO}

O objetivo deste trabalho é desenvolver o tema da Cultura da Paz, como fator indispensável e base do sucesso do novo paradigma constitucional da justiça no México, que ocorreu como resultado das reformas constitucionais sobre direitos humanos do 11․ Junho de 2011, que afetam o sistema jurídico mexicano, tanto teoricamente como normativamente. Assim, a questão dos direitos humanos torna-se não apenas um modelo legal, mas um modo de vida. Na primeira parte do trabalho, um quadro teórico do sujeito é exposto, para depois estabelecer a internacionalização da cultura da paz e sua relação direta com a educação, concluindo com as abordagens existentes entre o tema da paz e os direitos humanos. A educação sobre direitos humanos e seu processo educacional também é desenvolvida, estabelecendo no final do trabalho as conclusões pessoais dos temas discutidos.

\section{PALAVRAS-CHAVE}

Justiça, cultura, direitos humanos, paz, educação.

\section{INTRODUCCION}

La reforma constitucional del 18 de junio del 2008 , en materia de procuración e impartición de justicia, aunada a la reforma constitucional en materia de derechos humanos del 11 de junio del 2011, representó para México un cambio de paradigma para todo el sistema jurídico. El operario del derecho, sea cual sea su posición, se enfrenta al reto de cambiar su dinámica en el ejercicio profesional, requiere de una nueva capacitación para asumir los retos de este nuevo paradigma jurídico, deberá ser capaz de desarrollar competencia en comunicación verbal y oratoria, así como integrar en su ejercicio profesional la cultura de la paz y el respecto a los derechos humanos. Como bien es sabido, dentro de los elementos rectores de los cambios dentro del sistema jurídico, los juicios orales y la justicia alternativa son y serán ejes fundamentales del nuevo modelo. Los métodos alternos de solución de conflictos son un modelo de justicia como lo establece la Carta Magna pronta y expedita, que permite a los ciudadanos construir el puente de comunicación que por algún motivo se ha roto y encontrar una solución pacífica y efectiva a sus diferencias con la ayuda de un profesional denominado mediador o conciliador. Dicho mecanismo permitirá no sólo descongestionar la pesada carga de trabajo que actualmente tienen los juzgados civiles, familiares, mercantiles y penales, sino que permitirá que los ciudadanos eviten verse involucrados en conflictos de toda índole si logramos construir una CULTURA DE LA PAZ entre todos los miembros que conformamos esta sociedad.

De conformidad con los números que arroja el Instituto de Investigaciones Jurídicas del Congreso de la Unión, los métodos alternos son el presente y futuro del nuevo Sistema de Justicia en México, se estima que el $80 \%$ de los asuntos que actualmente se ventilan en los juzgados podrán y deberán resolverse por mecanismos alternativos, lo que representa una gran responsabilidad para todos y cada uno de los operadores del nuevo sistema.

El presente trabajo más que describir el modelo de justicia alternativa, tiene como objetivo desarrollar el tema de la Cultura de la Paz como un factor indispensable y base del éxito de este modelo de justicia, que deberá convertirse en un modelo de vida. En la primera parte del trabajo se expone un marco teórico del tema para, posteriormente, 
establecer la internacionalización de la cultura de la paz y su relación directa con la educación, concluyendo con las aproximaciones existentes entre el tema de la paz y los derechos humanos, desarrollando los aspectos que se relacionan con la educación en materia de derechos humanos y su proceso educativo, estableciendo al final del trabajo las conclusiones personales de los temas tratados.

\section{METODOLOGÍA}

Por la importancia que cobra la sistematización metodológica en la construcción del presente trabajo, estimamos conveniente recordar aquellas consideraciones que hizo el Lic. José Montes de Oca y Silva ${ }^{1}$ acerca de la investigación social, precisamente porque interesa conocer cuál es la relación que existe entre el Derecho constitucional y la sociedad mexicana contemporánea, siendo dicha vinculación el objeto cognoscitivo, respecto del cual centraremos nuestra atención."...En las investigaciones sociales es necesario emplear lo más ampliamente posible, la generalización, haciendo uso oportuno de la inducción y al mismo tiempo se requiere el empleo acertado del método de la comprensión de Dilthey, para poder descubrir el sentido o sentidos de toda acción del hombre, dado que éste es, por antonomasia un animal teleológico...." En mérito de lo anterior la metodología utilizada para describir, explicar y reflexionar sobre lo que es la cultura de la paz y su relación con el fenómeno social y jurídico, se basó en el uso de los métodos deductivo y descriptivo, los cuales sirvieron para analizar a través de los marcos teóricos abordados la definición del fenómeno de cultura de la paz, su interrelación en la vida jurídica y social del individuo y la importancia del tema en materia educativa, social y cultural, utilizando para lo anterior técnicas documentales e informáticas.

\section{MARCO TEÓRICO}

\subsection{Qué es la Cultura de la Paz}

El concepto más sencillo de paz, de conformidad con el diccionario de la lengua española, define a la misma simplemente como

1. Montes de Oca y Silva, José.- (1993) “Derecho Constitucional Mexicano", Editorial Porrúa, pp. 26. la ausencia de guerras; concepto centrado en los conflictos bélicos entre Estados².

En 1941, Wright estableció que la paz era un equilibrio dinámico de factores políticos, sociales, culturales y tecnológicos y al romperse la "armonía" en el sistema internacional venía el conflicto bélico. ${ }^{3}$ El concepto de paz fue evolucionando, no sólo incluyó guerras entre países sino también aterrizó a la violencia en las casas, esta idea según algunos autores, contempla a la guerra como una forma masculina de afrontar los conflictos, por lo cual dicha corriente recibió el nombre de Paz femenina ${ }^{4}$.

El sociólogo y matemático Johan Galtung, la dividió en dos categorías: paz negativa (no guerra) y paz positiva (no violencia) ${ }^{5}$. Según esta división la primera se refiere a la ausencia de violencia personal, guerras, terrorismo y disturbios mientras que la segunda se da cuando existe una ausencia de violencia estructural, esto es, ausencia de pobreza, hambre, discriminación y contaminación ${ }^{6}$.

El citado autor señala que la contraposición de la paz no es la guerra sino la violencia, de ahí que cuando tratemos de entender lo que es la paz, debemos comenzar por el estado de ausencia o disminución de todo tipo de violencia, tanto directa (física y verbal), estructural o cultural, que vaya dirigida tanto a mente, cuerpo o espíritu de cualquier humano o contra la naturaleza ${ }^{7}$.

Posteriormente, ya en los años 90, surge el planteamiento de la Paz Holística-Gaia, dándole un alto valor a la relación entre los seres humanos y el medio ambiente. Para mediados de esa década surge la teoría de la Paz Holística interna y externa, que incluye también aspectos espirituales ${ }^{8}$.

2. Fisas, Vinces. Cultura de paz y gestión de conflictos. Ed. Icaria/ Antrazyt. UNESCO. Barcelona 2001. p.21

3. Idem

4. Reardon, Betty. Militarization, security and peace education. United ministres in education, USA, 1982.

5.Infra. Capítulo 1

6. Aiello de Almeida, María Alba, Mediación: Formación y algunos aspectos claves. Ed. Porrúa. México, 2001. p. 7

7. Fisas, Vinces. Op. Cit. P. 19

8. Idem. 
Por su parte, el investigador colombiano Díaz Gamboa, asegura que la categorización de investigación científica sobre la paz ha abierto un escenario disciplinar de la mayor importancia,

La investigación sobre la paz ha sido elevada a actividad científica dirigida a estudiar las condiciones de eliminación de la guerra y en general de la violencia armada como métodos de conducción y resolución de los conflictos en una sociedad. Se trata de una investigación multidisciplinaria, pues en la misma se involucran la ciencia política, la sociología, las relaciones internacionales, la economía, la sicología, la historia, la filosofía, el derecho internacional, la estadística, las matemáticas, la demografía, etc 9 .

Al hablar de construir un entorno pacífico, necesariamente tenemos que contemplar los temas de conflicto, violencia y guerra, todos ellos materias de estudio y trabajo de los Métodos Alternos de Solución de Conflictos, además de ser medios de construcción y conservación de la paz al encontrar solución o prevenir las controversias. La herramienta principal del mediador es fomentar el diálogo, conduciéndose por el camino de la paz para llegar a la paz, ${ }^{10}$ con el propósito de fomentar la empatía, la no violencia y la creatividad que potencie las competencias para superar el conflicto.

La mediación significa lograr una solución en consenso, a pesar de los problemas previos, esto es, una transformación de la fractura en una convivencia ${ }^{11}$.

Así lo explica Galtung al considerar a la transformación de conflictos como un medio para alcanzar la paz y explicarla como "aquello que tenemos cuando es posible transformar los conflictos en forma creativa y no violenta". ${ }^{12}$

9. Díaz Gamboa, Luis, Nuevas configuraciones del Derecho a la Paz. Revista Misión Jurídica. Ed. Universidad Colegio Mayor de Cundinamarca. Bogotá. No. 9. 2015. p. 260.

10. El construir la paz a partir del establecimiento de un ambiente de paz, es una idea que ya podíamos encontrar en textos como la Carta del Apóstol Santiago 3:16. donde se afirmaba que "Fruto de justicia, ella se siembra en paz, para bien de los que siembran la paz."

11. Gottheil, Julio. "La mediación y salud del tejido social". En Gottheil, Julio y Schiffrin, Adriana (comp.) Mediación: una transformación de la cultura. Ed. Paídos. Buenos Aires 1996. p. 215-225.

12. Galtung, Johan. Peace by Peaceful Jeans. Peace and Conflict,
Transformar el conflicto significa trascender los objetivos de las partes involucradas en éste, definiendo otros objetivos, y saliendo del discurso inmovilista. ${ }^{13}$ Es claro que dicho proceso de transformación se presenta en la mediación, ya que al tercero neutral e imparcial se constituye como un verdadero facilitador para la solución de conflictos: un real trabajador de paz. Johan Galtung establece la idea de la transformación de los conflictos como medio para lograr la paz en su teoría de las tres Rs, compuesta de tres elementos: (i) Reconstrucción (tras la violencia), (ii) Reconciliación (de las partes) y (iii) Resolución ${ }^{14}$.

El propio Galtung relaciona las actitudes, conducta y contradicción con tres manifestaciones de violencia: directa, cultural y estructural, vinculando la reconstrucción con la recuperación tras la violencia directa y la resolución de las contradicciones e incompatibilidades tratando de superar la violencia estructural.

Cabe destacar que la teoría de Galtung tiene como fundamento, en muchos sentidos, enseñanzas de diferentes religiones, que contienen algunos elementos importantes para la construcción de una cultura de paz ${ }^{15}$.

Perspectiva hindú: Existe un conflicto destructivo y un conflicto creador, uno como fuente de violencia y otro del desarrollo. El mediador actúa como un preservador, trasmutando la violencia y fomentando el desarrollo.

Perspectiva budista: Los conflictos no empiezan ni terminan, todo crece por una causa y en ella tenemos parte de responsabilidad.

Perspectiva cristiana: La responsabilidad en la transformación de los conflictos está vinculada con los individuos, con sus decisiones, su promoción de la paz o la violencia.

Perspectiva taoísta: El todo es bueno y malo, yin y yang, existen muchas posibilidades de que la

\footnotetext{
Development and Civilization. Ed. Prio, Oslo, 1996. p. 9

13. Fisas, Vinces. Op. Cit. P. 233.
}

14. Fisas, Vinces. Op. Cit. P. 231

15. Galtung, Johan. "Conflict transformación by peaceful jeans",www.transcendt, 23 de marzo del 2016 
elección tomada tenga consecuencias negativas y que la acción no elegida tenga positivas, por ello es importante hacer sólo lo que puede deshacerse.

Perspectiva islámica: La responsabilidad debe ser sometida a un propósito común para lograr el bienestar de todos.

Perspectiva judaica: La verdad está menos en la fórmula que en el diálogo requerido para buscar la fórmula, dicho diálogo no tiene principio ni fin. ${ }^{16}$

El escritor estadounidense John Paul Lederach pone especial importancia en la reconciliación para poder lograr la construcción de la paz. Según dicho autor la reconciliación representa un lugar, el punto de encuentro donde se pueden aunar los intereses del pasado y del futuro. ${ }^{17}$ Por ello, es necesario que las partes en conflicto encuentren formas para encontrarse consigo mismas y con sus enemigos, tomando en cuenta sus necesidades y miedos.

En criterio de Galtung, la reconciliación está constituida de dos momentos: a) El cierre, es decir, que no se regrese a las agresiones, para lo que es fundamental la conducta y b) La curación, esto es, el proceso de rehabilitación, en el que se base el cambio de actitud. ${ }^{18}$ La reconciliación es un proceso implícito en la transformación de los conflictos, ayuda a los involucrados a superar sus traumas y problemas. ${ }^{19} \mathrm{La}$ reconciliación es una potencialidad ya que ofrece posibilidades que se construyen sobre mecanismos que comprometen a las partes en conflicto, las acercan, las ponen de frente y logran hacer que se acepten. La reconciliación es un espacio de verdad, misericordia, justicia y paz. ${ }^{20}$

16. Galtung, Johan. Tras la violencia, 3R: reconstrucción, reconciliación, resolución. Ed. Bakeaz/Germina Gogoratuz. Bilbao 1998. p. 18

17. Lederach, John Paul. Construyendo la paz. Reconciliación sostenible en sociedades diversas. Ed. Bakeaz. Bilbao 1998. p. 47

18. Romera I Rueda, Raul. Guerra, posguerra y paz. Pautas para el análisis y la intervención en contextos posbélicos o postacuerdo. Ed. Icaria. Barcelona 2003. p. 49

19. Lederach, John Paul. Op. Cit. P. 47

20. Ibidem

\section{2.- Que se entiende por Educar para la paz}

Iniciamos el presente tema describiendo lo que para el político francés Jacques Delors, tiene como propósito la educación , citando al autor : “ La educación tiene el propósito de instruir a cada individuo para desarrollar todas sus capacidades al máximo, realizando su potencial creativo, incluyendo la responsabilidad de sus propias vidas, así como el cumplimiento de sus objetivos personales"21. Por su parte, Vinces Fisas menciona que la educación es un instrumento crucial de la transformación social y política. Si entendemos a la paz como la transformación creativa de los conflictos, teniendo como elementos clave, el conocimiento, la imaginación, la comprensión, el diálogo, la solidaridad, la integración, la participación y la empatía ${ }^{22}$ podemos estar de acuerdo en que su propósito no es otro que formar una cultura de paz, ${ }^{23}$ opuesta a la cultura de la violencia que se padece en la actualidad, donde se puedan desarrollar valores, competencias y potencialidades ${ }^{24}$. Por otra parte, el político español Federico Mayor Zaragoza, establece que por educación para la paz se entiende el proceso de participación por el cual debe desarrollarse la capacidad crítica de las personas, elemento esencial de lo que se pretende sean los nuevos ciudadanos ${ }^{25}$.

21. Delors, Jacques. Educación: Hay un tesoro escondido dentro. Ed. UNESCO, España. 1996. p 250

22. Cfr. Pérez Sauceda, José Benito, Zaragoza Huerta José y Barba Alvarez, Rogelio. "La interdisciplinariedad y multidisciplinariedad comomodelos a seguir en la enseñanza del Derecho: La experiencia de los Métodos Alternos de Solución de Controversias" en Letras Jurídicas: revista electrónica de Derecho. Universidad de Guadalajara. Primavera del 2009. N. 8 http://letrasjuridicas.cuci.udg.mx/numeros/articulos8/ La\%20interdisciplinariedad\%20y\%20multidisciplinariedad pdf V. Página de la Revista: http://letrasjuridicas.cuci.udg.mx/

23. El Preámbulo Fundacional de la Constitución de Organización de las Naciones Unidas para la Educación, la Ciencia y la Cultura (UNESCO) inicia con la siguiente frase: "Puesto que la guerra nace en la mente de los hombres, es en la mente de los hombres donde deben erguirse los baluartes de la paz" Creo que no hay un mejor enunciado que pueda explicar el objetivo de la educación como medio para lograr la transformación de la sociedad del conflicto en que vivimos, a la sociedad de la paz, que ponga en práctica el verdadero conocimiento, el del bien común. V. Constitución de Organización de las Naciones Unidas para la Educación, la Ciencia y la Cultura-Preámbulo Fundacional de la Constitución de Organización de las Naciones Unidas para la Educación, la Ciencia y la Cultura.

24. Fisas, Vinces. Op. Cit. P.374.

25. Mayor Zaragoza, Federico "Educación para la Paz" en Educación XXI: Revista de la Facultad de Educación. Ed. UNED. Madrid, No. 6. 2003. p. 19 
Así pues la educación para la paz es el análisis del mundo en que vivimos mediante reflexiones críticas emanadas de valores, en una cosmovisión pacifista, que convierta al individuo en un ente transformador, liberador de personas, obligado por la conciencia para cooperar en la emancipación de todos los seres humanos y de sí mismo ${ }^{26}$.

Educar para la paz es más que la trasmisión de conocimientos, es un cambio de actitudes, hábitos $\mathrm{y}$ valores, considerando a éstos, como proyectos globales de existencia, que generan predisposiciones que el individuo va interiorizando y asumiendo progresivamente ${ }^{27}$.

Educar para la paz es un luchar contra la pereza y la tendencia al conformismo y el silencio que la sociedad fomenta ${ }^{28}$. Es dotar a la persona para razonar y decidir con libertad. Significa establecer directrices para poder defender nuestras diferencias y divergencias sin violencia. ${ }^{29}$

La Educación para la Paz es una responsabilidad y un proceso global de la sociedad, en donde las personas y los grupos aprenden a desarrollar conscientemente la totalidad de sus capacidades, actitudes, aptitudes y conocimientos, en el interior de sus comunidades y para beneficio de las mismas ${ }^{30}$ con el fin de crear y vivir una Cultura de Paz. ${ }^{31}$

26. Rodríguez, Martín. "Educar para la paz y la racionalidad comunicativa", en Educando para la Paz: Nuevas Propuestas. Universidad de Granada, España. 1994. p. 366.

27. Aguilera Portales, Rafael. "La Mediación. Un acercamiento real a la Justicia y a la Cultura de Paz" En Gorjón Gómez, Francisco Javier, et al. Mediación y Arbitraje. Leyes Comentadas y Concordadas del Estado de Nuevo León. Ed. Porrúa. México. 2009. p. 51-68.

28. Mayor Zaragoza, Federico. Op. Cit. P. 19.

29. Fisas, Vinces. Op. Cit. P. 372.

30. Para Jacques Delors, son cuatro los conocimientos basados en una Educación para la Paz que debe conocer toda persona: a) Aprender a conocer: Adquiriendo los instrumentos de comprensión; b) Aprender a Hacer: Para poder actuar en el entorno; c) Aprender a vivir juntos: Para participar y cooperar con los demás en las actividades sociales d) Aprender a ser: Elemento en que funcionan los tres anteriores. Idem.

31. Sánchez Aneas, Asela. Acoso escolar y convivencia en las aulas. Manual de prevención e intervención. Ed. Formación Académica. España. 2009. p. 449.
Los objetivos que se buscan lograr mediante la educación para la paz y la no violencia son los siguientes: ${ }^{32}$

La concienciación y sensibilización de la comunidad educativa acerca de la importancia de una adecuada convivencia escolar y los medios que existen para mejorarla.

La promoción de una Cultura de paz en los centros educativos y el mejoramiento de la convivencia escolar, facilitando el diálogo y la participación real de todos los sectores de la comunidad educativa.

El fomento de los valores, actitudes y las prácticas que permitan mejorar el cumplimiento de las normas, el avance del respeto a la diversidad cultural, la promoción de la igualdad entre hombres y mujeres, en la prevención, detección $\mathrm{y}$ tratamiento de manifestaciones violentas, especialmente la violencia de género y las actitudes y comportamientos xenófobos y racistas.

\subsection{La cultura de la paz en el ordenamiento internacional}

\subsection{1 la cultura de la paz en UNESCO}

El 16 de noviembre de 1945, se funda la Organización de las Naciones Unidas para la Educación, la Ciencia y la Cultura (UNESCO) que tiene entre otros propósitos fomentar el diálogo entre las civilizaciones, las culturas, los pueblos basados en el respeto, una de las bases fundamentales de la educación y la cultura de paz. ${ }^{33}$

La Educación para la Paz, debe apreciar los siguientes aspectos:

- Democracia

- Justicia

- Desarme

- Derechos Humanos

- Tolerancia

- Respeto a la Diversidad Cultural

- Preservación del Medio Ambiente

- Prevención y Resolución de Conflictos

- Reconciliación

32. Ídem.

33. Página de la UNESCO. http://portal.unesco.or/es/ev.php-

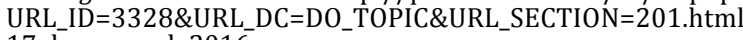
17 de marzo de 2016 
- No Violencia

- Teoría de la Paz

Actualmente el tema recibe un importante soporte teórico y práctico, tanto en centros docentes de diversos niveles de aprendizaje, así como en centros de investigación e instituciones y organismos internacionales.

En 1994, la UNESCO instauró la Unidad del Programa de Cultura de Paz con el objetivo de perfeccionar las metodologías para favorecer y fortalecer la reflexión, la investigación y la evaluación sobre la Cultura de Paz. En 1995 la misma organización adopta la resolución 5.3 del Proyecto transdisciplinario: Hacia una Cultura de Paz en el que se implementan 3 ejes que son: ${ }^{34}$ (i) La Cultura de Paz; (ii) La Educación para la Paz y (iii) La Cultura de Paz en la práctica.

La misma UNESCO desarrolla el proyecto "Estrategias a Plazo Medio 1996-2001 para contribuir a la consolidación de la paz" basado en 4 grandes apartados: ${ }^{35}$

1. 1-Fomentar la Educación para la Paz, los Derechos Humanos, la Democracia, la Tolerancia $y$ el entendimiento internacional.

2. 2-Promover los Derechos Humanos y la lucha contra la discriminación.

3. 3-Apoyar la consolidación de los procesos democráticos.

4. 4-Contribuir a la Prevención de Conflictos y a la Consolidación de la Paz una vez terminados los conflictos.

De igual forma en reuniones mundiales se han desarrollado los lineamientos a seguir como las sucedidas en Montreal y Viena en 1993 y la Haya en 1997, hasta llegar al punto cúspide que es la aprobación unánime de la Declaración y el Programa de Acción para una Cultura de Paz del 13 de septiembre de 1999 de la que hablaremos más adelante. Posteriormente se realizaron acciones como el "2000, Año Internacional para una Cultura de Paz" y la declaración del Decenio 2001-2010 de una Cultura de Paz y No Violencia

34. Fisas, Vinces. Op. Cit. P. 371

35. Idem. para los niños del mundo por parte de las Naciones Unidas ${ }^{36}$.

La UNESCO ha contribuido enormemente al desarrollo de la Educación para la Paz, mediante la promoción de revistas, editoriales, colecciones y programas sobre la paz, la creación de las Cátedras UNESCO sobre Cultura de Paz, ${ }^{37}$ así como el desarrollo, introducción y práctica de métodos tradicionales de resolución de conflictos (como la Mediación), el entrenamiento de "promotores de paz" para la prevención, transformación y resolución de conflictos, la formación de Mediadores/Conciliadores, así como la capacitación y práctica de solución de conflictos y Mediación en el sistema escolar, ${ }^{38}$

Como podemos advertir, la propia UNESCO entiende que la alternativa a la cultura de la no violencia debe sustentarse en la negociación, el diálogo, la mediación, el empoderamiento, la empatía, y la capacidad de manejar nuestros propios conflictos, seguramente podremos aprender mucho de cuanto se ha dicho y hecho al respecto en gestión, resolución o transformación de conflictos ${ }^{39}$.

El proyecto de construir una cultura de paz en buena medida no es otra cosa que el reto planetario de abordar los conflictos desde otra mirada, con otras herramientas y con otros propósitos, que comienza por la Educación de la $\mathrm{Paz}^{40}$.

La Cultura de Paz y No violencia, ${ }^{41}$ tiene como base la aceptación de que las personas tienen

\section{Ibidem}

37. En este sentido la Línea de Investigación de Métodos Alternos de Solución de Controversias del Centro de Investigación de Tecnología Jurídica y Criminológica 8CITEJYC) de la Universidad Autónoma de Nuevo León (UANL), dentro de sus proyectos está el desarrollo e impartición de una Cátedra UNESCO de MASC y Cultura de Paz

38. Fisas, Vinces. Op. Cit. P. 384-385.

39. Fisas, Vinces. Op. Cit. P. 183. www.find-culturadepaz.org.

40. Idem

41.La No Violencia como su nombre lo indica, es una situación o una acción sin violencia, ausente de violencia, es lo opuesto a conflicto y a la violencia. V. Zaragoza Huerta, José, Núñez Torres, Michael y Pérez Sauceda, José Benito. "La No violencia y el Derecho" en Revista Ciencia, Conocimiento y Tecnología Número 82, Ed. Coordinación de Ciencia y tecnología de Nuevo León, Monterrey, N. L., México, 2008. p. 46-47 y Patfoort, Pat. Erradicar la Violencia. Construyendola No Violencia.Ed. 
derecho a la Paz, lo cual implica un rechazo a la violencia y la aceptación de la gestión de conflictos mediante el diálogo y la negociación. Por ello, la educación en la convivencia social y en la resolución pacífica de conflictos es indispensable para lograr dicha cultura. ${ }^{42}$

\subsection{2- Los Ejes de la Escuela de Paz y la resolución de conflictos ${ }^{43}$}

La educación para la paz busca fomentar competencias en solucionar conflictos, en resolver guerras, en prevenir la violencia, en impedir todo aquello que es contrario a la vida y a la dignidad humana, constituyendo al ser como un elemento para favorecer la transformación de la realidad de una cultura de guerra a una de paz ${ }^{44}$. La Educación para la paz con enfoque en la mediación y la resolución de conflictos, se centra en el análisis de las controversias, desde el personal hasta el global, con el objetivo de solucionarlos sin violencia. En conclusión, debemos formar a las personas para ver los conflictos con ojos de pacificador, con la mente en pos de la solución ${ }^{45}$,

Lumen. Buenos Aires. 2004. p. 83. Para Gandhi, la No violencia es el rechazo moral de la violencia y la convicción de una vía alternativa para manifestarse. Sin embargo, el ser no violento no implica rehusar el conflicto, en palabras de Gandhi: "Ningún hombre puede ser activamente no violento si no se rebela contra las injusticias sociales donde quiera que se den". La No violencia solamente rechaza el conflicto como medio para solucionar los conflictos. V. Apud. Corral Prieto. La No Violencia. Historias y perspectivas. Ed. CCS. Madrid. 1993. p. 26.

42. Sánchez Aneas, Asela. Op. Cit. P. 447.

43.La Escuela de Paz es un proyecto basado en la Educación para la Paz, implica a toda la comunidad educativa en un proceso de aprendizaje, donde se aplican estrategias para mejorar la convivencia y la prevención de conflictos o su resolución pacífica. V.Sánchez Aneas, Asela. Op. Cit. P. 453. En lo referente a la importancia de las competencias comunicativas, la Mediación y la Cultura de Paz, la Dra. Beatriz Ferrero establece: El ser humano es un ente social, la comunicación es inherente a él, siendo indispensable para desarrollo de relaciones interpersonales, que en caso de fracaso puede que se recurra a la violencia para solucionar sus diferencias. Es en este sentido, donde puede hablarse de la contribución de la Mediación a una Cultura de Paz con base en el concepto de racionalidad comunicativa de Haberlas, que permitiría establecer, o en su caso restablecer, los lazos sociales a través del diálogo". V. Fernández Herrero, Beatriz. "La Dimensión Formativa de la Mediación: Educación Moral y Ciudadana" en Educaweb. Sitio en la red de Educación y formación de España. Publicado el 29 de Junio de 2009. http://www.educaweb.com/noticia/2009/06/29dimensionformativa-mediacion-educacion-moral-ciudadana-13745.html

44. Fisas, Vinces. Op. Cit. p. 182.

45. Como lo establece Gandhi, uno de los más grandes promotores de los movimientos no Violentos: "La Ciencia de la Guerra nos conduce a la Dictadura pura y dura". Sólo la Ciencia de la No Violencia puede llevarnos a una auténtica democracia. pues seguramente si buscamos la paz mediante el diálogo y la cooperación, la podremos conseguir.

\subsubsection{La Cultura de la Paz en la ONU}

Reconociendo que la paz no sólo es la ausencia de conflictos, sino que también requiere un proceso positivo, dinámico y participativo en que se promueva el diálogo y se solucionen los conflictos en un espíritu de entendimiento y cooperación mutuos, el 6 de octubre de 1999, la Asamblea General de las Naciones Unidas aprueba la resolución 53/243 “Declaración y Programa de Acción sobre una Cultura de Paz", con el fin de promover que los gobiernos, las organizaciones internacionales y la sociedad civil orienten sus actividades al fomento y promoción de una cultura de paz en beneficio de los pueblos del mundo.

En dicha declaración se define a la cultura de paz, como un conjunto de valores, actitudes, tradiciones, comportamientos y estilos de vida basados en:

El respeto a la vida, el fin de la violencia y el fomento y la práctica de la no violencia por medio de la educación, el diálogo y la cooperación;

El respeto pleno de los principios de soberanía, integridad territorial e independencia política de los Estados y de no injerencia en los asuntos que son esencialmente jurisdicción interna de los Estados, de conformidad con la Carta de las Naciones Unidas y el derecho internacional;

El respecto pleno y la promoción de todos los derechos humanos y las libertades fundamentales;

El compromiso con el arreglo pacífico de los conflictos;

Los esfuerzos para satisfacer las necesidades de desarrollo y protección del medio ambiente de las generaciones presentes y futuras;

El respeto y la promoción del derecho al desarrollo;

El respeto y el fomento de la igualdad de derechos y oportunidades de mujeres y hombres;

V. Gandhi, Mohandas. Escritos Esenciales. Ed. Sal Térrea. España. 2004. p. 194 
El respecto y el fomento del derecho de todas las personas a la libertad de expresión, opinión e información;

La adhesión a los principios de libertad, justicia, democracia, tolerancia, solidaridad, cooperación, pluralismo, diversidad cultural, diálogo y entendimiento a todos los niveles de la sociedad y entre las naciones; animados por un entorno nacional e internacional que favorezca a la paz ${ }^{46}$.

Para lograr esta cultura se requieren el desarrollo de valores, actitudes, comportamientos $\mathrm{y}$ estilos de vida que sean propensos al fomento de la paz tanto individual, social como entre las naciones.

De acuerdo con la Declaración, los elementos que se necesitan para el pleno desarrollo de una cultura de paz son:

La promoción del arreglo pacífico de los conflictos, el respeto y el entendimiento mutuo y la cooperación internacional;

El cumplimiento de las obligaciones internacionales contraídas en virtud de la Carta de las Naciones Unidas y el derecho internacional;

La promoción de la democracia, el desarrollo de los derechos humanos y las libertades fundamentales y el respeto y cumplimiento universales de éstos;

La posibilidad de que todas las personas a todos los niveles desarrollen aptitudes para el diálogo, la negociación, la formación de consenso y la solución pacífica de controversias;

El fortalecimiento de las instituciones democráticas y la garantía de la participación plena en el proceso del desarrollo;

La erradicación de la pobreza y el analfabetismo y la reducción de las desigualdades entre las naciones y dentro de ellas;

La promoción del desarrollo económico y social sostenible;

La eliminación de todas las formas de discriminación contra la mujer promoviendo su

46. V. Artículo 1 de la Declaración y Programa de Acción sobre una Cultura de Paz de la Organización de las Naciones Unidas. autonomía y una representación equitativa en todos los niveles de la adopción de decisiones;

El respeto, la promoción y la protección de los derechos del niño;

La garantía de la libre circulación de información en todos los niveles y la promoción del acceso a ella;

El aumento de la transparencia y la rendición de cuentas en la gestión de los asuntos públicos;

La eliminación de todas las formas de racismo, discriminación racial, xenofobia e intolerancia conexas;

La promoción de la comprensión, la tolerancia y la solidaridad entre todas las civilizaciones los pueblos y las culturas, incluso hacia las minorías étnicas, religiosas y lingüísticas;

El respeto pleno del derecho a la libre determinación de todos los pueblos, incluso aquellos que viven bajo la dominación colonial $u$ otras formas de dominación u ocupación extranjera, como lo consagra la Carta de las Naciones Unidas y está expresado en los Pactos Internacionales de Derechos Humanos, así como en la Declaración sobre la concesión de la independencia a los países y pueblos coloniales contenida en la resolución 1514 (XV) de la Asamblea General, de 14 de diciembre de $1960^{47}$.

La Declaración para la Cultura de Paz remarca el papel fundamental de la educación para poder alcanzar un estado de paz, sobre todo la formación social en derechos humanos ${ }^{48}$. Para ello es muy importante el rol que juegan los gobiernos, la sociedad civil, las organizaciones no gubernamentales y los medios de comunicación ${ }^{49}$.

47. V. Artículo 3 de la Declaración y Programa de Acción sobre una Cultura de Paz de la Organización de las Naciones Unidas.

48. V. Artículo 4 de la Declaración y Programa de Acción sobre una Cultura de Paz de la Organización de las Naciones Unidas.

49. En este sentido, para Asela Sánchez, son tres los conceptos que interactúan entre sí, en los que se basa la Cultura de Paz y son: a) La Paz Positiva; b) El Desarrollo humano sustentable y c) La democracia participativa, es decir, precisamente una sociedad en la que todos sus integrantes participan activamente en la realización de sus propósitos. V. Sánchez Aneas, Asela. Op. Cit. p. 448 
La Declaración sobre una Cultura de Paz no es un intento fuera de la realidad o inalcanzable, sin tareas específicas para poder lograr el Estado de Paz que se pretende. La Declaración contiene un Programa de acción con lineamientos claros en los que la Organización de las Naciones Unidas y la UNESCO se comprometen a apoyar.

El programa de Acción sobre una Cultura de Paz se divide en dos apartados, que contienen a su vez planteamientos específicos y son:

a). Objetivos, Estrategias y Agentes Principales:

1. El Programa constituye la base para lo que fue el Año Internacional de la Cultura de la Paz y el Decenio Internacional de una cultura de paz y no violencia para los niños del mundo;

2. Se alienta al os Estados Miembros de las Naciones Unidas a adoptar medidas para el fomento y promoción de una cultura de paz en el plano nacional, así como en los planos regional e internacional;

3. La participación de la sociedad civil en los planos local, regional y nacional con el objetivo de ampliar el ámbito de las actividades relativas a una cultura de paz;

4. Las Naciones Unidas deben reforzar las actividades que realiza a favor de una cultura de paz;

5. La Organización de las Naciones Unidas para la Educación, la Ciencia y la Cultura mantendrá su función esencial en la promoción de una cultura de paz, contribuyendo a dicho propósito;

6. El apoyo y consolidación de las asociaciones entre los diversos agentes que se indican en la Declaración con el fin de provocar un movimiento mundial a favor de una cultura de paz;

7. La cultura de paz se promueve mediante el intercambio de información entre los agentes sobre sus iniciativas a este respecto;

8. La ejecución eficaz del Programa de Acción exige la movilización de recursos, incluidos financieros, por parte de los gobiernos, las organizaciones y los particulares interesados.

b). Consolidación de las medidas que adopten todos los agentes pertinentes en los planos nacional, regional e internacional:
9. Medidas para promover una cultura de paz por medio de la educación;

10. Medidas para promover el desarrollo económico y social sostenible;

11. Medidas para promover el respeto de todos los derechos humanos;

12. Medidas para garantizar la igualdad entre mujeres y hombres;

13. Medidas para promover la participación democrática;

14. Medidas encaminadas a promover la comprensión, la tolerancia y la solidaridad;

15. Medidas destinadas a apoyar la comunicación participativa y la libre circulación de información y conocimientos;

16. Medidas para promover la paz y la seguridad internacionales ${ }^{50}$.

\section{LOS DERECHOS HUMANOS Y LA CULTURA DE LA PAZ}

Los derechos humanos constituyen uno de los pilares fundamentales de la cultura de paz, pues al promover la comprensión, el respeto y la solidaridad, es posible lograr una convivencia más justa, libre y respetuosa de la dignidad humana. Educar en materia de derechos humanos es una tarea compleja que, si bien requiere el compromiso de las autoridades, aún más el de la sociedad civil, pues se requiere del apoyo de todas $\mathrm{y}$ todos para generar cambios estructurales que faciliten la convivencia en un contexto de plena vigencia de los derechos humanos. Consolidar la cultura de paz es un trabajo arduo, el cual requiere de la intervención de diversos actores, tanto públicos como privados, que se esfuercen por el arreglo pacífico de los conflictos, el respeto y el entendimiento mutuos, la promoción de la democracia, la plena vigencia de los derechos humanos y las libertades, la construcción del diálogo, la negociación, la búsqueda de consenso, la promoción del desarrollo económico y social, la eliminación de toda forma de discriminación, entre otras muchas acciones.

Como dijo Eleonor Rooselvet: “"No basta con hablar de paz. Uno debe creer en ella y trabajar para conseguirla".

50. ONU. Acción sobre una Cultura de Paz, Resolución 53/243. 1999. 


\subsection{La enseñanza de los derechos humanos en la cultura de la paz}

La primera referencia contemporánea a la enseñanza de los derechos humanos la encontramos en la propia Carta de las Naciones Unidas de 1945 y, más concretamente, en la Declaración Universal de los Derechos Humanos de 1948, en cuyo preámbulo se dice que la Asamblea General de las Naciones Unidas proclama la Declaración como ideal común "por el que todos los pueblos y naciones deben esforzarse, a fin de que tanto los individuos como las instituciones, inspirándose constantemente en ella, promuevan, mediante la enseñanza y la educación, el respeto a estos derechos y libertades". Luego, en su Artículo 26, inciso 2, subraya que

La educación tendrá por objeto el pleno desarrollo de la personalidad humana y el fortalecimiento del respeto a los derechos humanos y a las libertades fundamentales; favorecerá la comprensión, la tolerancia y la amistad entre todas las naciones y todos los grupos étnicos o religiosos; y promoverá el desarrollo de las actividades de las Naciones Unidas para el mantenimiento de la paz.

La Unesco ha asumido el liderazgo internacional en el campo de la enseñanza de los derechos humanos. La Asamblea General de las Naciones Unidas resolvió el año 1968 pedir a sus Estados Miembros que tomaran, cuando conviniera, medidas para introducir o estimular, según el sistema escolar de cada Estado, los principios proclamados en la "Declaración Universal de Derechos Humanos" y otras declaraciones. Pidió, asimismo, la enseñanza progresiva de esta materia en los programas de las escuelas primarias y secundarias.

En la Décima Octava Conferencia General de la Unesco, celebrada en París en 1974, los Estados Miembros de la organización aprobaron la "Recomendación sobre la educación para la comprensión, la cooperación y la paz internacionales y la educación relativa a los Derechos Humanos y las libertades fundamentales". En virtud de dicha recomendación, los Estados adquirieron el compromiso de introducir, en todos los niveles y modalidades del sistema educativo, la enseñanza de los derechos humanos. En 1993 se celebró en Viena, Austria, la Conferencia Mundial sobre los Derechos Humanos, que aprobó el "Plan Mundial de Acción sobre educación para los Derechos Humanos y la Democracia". Dicho Plan, reafirmó que "los valores democráticos son requeridos para el disfrute de los derechos humanos y las libertades fundamentales, por lo cual la educación sobre los derechos humanos y la democracia deberían recibir atención especial". El Plan incluso sostiene que "la educación sobre los derechos humanos y la democracia es en sí misma un derecho humano, y un prerrequisito para la plena realización de la justicia social, la paz y el desarrollo". De esta suerte, el Plan eleva a la categoría de derecho humano el derecho a recibir una educación sobre los derechos humanos y la democracia. Ergo, cuando un gobierno desatiende este tipo de educación, está violando un derecho humano, que concierne especialmente a los niños y a los jóvenes, pero que pertenece a todas las personas, sin límite de edad.

El respeto a los Derechos Humanos es la base para construir una Cultura de Paz, paradigma adoptado por las Naciones Unidas como guía del Siglo XXI. La Cultura de Paz se funda en los valores universales de respeto a la vida, la libertad, la justicia, la solidaridad, la tolerancia y la igualdad de género. Podemos concluir que los Derechos Humanos y la Cultura de Paz deben ser los ejes de la educación para el siglo XXI.

\section{2.- El derecho humano a la paz}

Muchos pensadores han considerado que la paz es un anhelo universalmente reconocido que ha sido expresado e ilustrado a lo largo de la historia. Encontramos el primer pensamiento racional acerca de la paz casi simultáneamente en Oriente y en Occidente, en China y en Grecia; las propuestas chinas de desarme datan de 546 a. C. y son paralelas a los intentos griegos de usar alianzas para terminar con las guerras internas y contener las externas. Pero si bien ese deseo pudo surgir en un mismo momento inspirado por la necesidad de acabar con los desastres y con el imperio de la violencia, no alcanzó hasta fechas muy recientes un consenso en cuanto a su definición y realización práctica. Así, a lo largo de la línea del tiempo nos encontramos distintas versiones de "Paz" - eire griego; pax romana; santhi, hinduista; ahimsa, jainista; la paz, taoista; shalom, hebreo; pax hispánica; pax americana, etc. - que representan diferentes modos de concebir y organizar el mundo, así como de resolver y enfrentar los conflictos. Concepciones 
de paz negativa, como ausencia de guerra, o de paz positiva, como construcción de la justicia social. Polarización que persiste en la actualidad y que impide un futuro mejor para las próximas generaciones.

Al ser la paz un derecho humano corresponde el deber de su construcción a todos los seres humanos, aun cuando ese edificio jamás pueda terminarse definitivamente. Y esto porque la paz es un proceso que implica una forma de relación de los seres humanos entre sí y a través de las distintas formas de organización social que excluye la violencia en todas sus manifestaciones. Por otra parte, se inicia con el reconocimiento del derecho de los demás a una vida digna, se lleva adelante a través del diálogo y, por último, necesita de la cooperación. Para que se establezca un diálogo genuino es necesario que se acepte la necesidad de dialogar, que exista la voluntad de comprensión mutua y que las concesiones sean equilibradas. El Congreso Internacional sobre "La paz en el espíritu de los hombres", celebrado en el verano de 1989 en Yamoussoukro, Costa de Marfil. La Declaración surgida de este congreso trata de superar las distintas concepciones elaboradas (paz como ausencia de guerra, paz como equilibrio de fuerzas en el sistema internacional, paz negativa y paz positiva, paz holística, paz feminista...) al considerar que: 1/ La paz es esencialmente el respeto de la vida; 2/ La paz es el bien más precioso de la humanidad; 3/ La paz es más que el fin de los conflictos armados; 4/ La paz es un comportamiento; 5/ La paz es una adhesión profunda del ser humano a los principios de libertad, justicia, igualdad y solidaridad entre todos los seres; 6/ La paz es también una asociación armoniosa entre la humanidad y la naturaleza.

El futuro exige más que nunca la construcción de la paz, a través de la ciencia, la cultura, la educación y la comunicación, debido a que el respeto al derecho humano a la paz inspirado en el ideal democrático de dignidad, igualdad y respeto de la persona es la vía más segura para luchar contra la exclusión, la discriminación, la intolerancia y la violencia que amenazan la cohesión de las sociedades y conducen a los conflictos armados. Por otro lado, nuevas amenazas pesan hoy sobre la seguridad internacional como las desigualdades insostenibles tanto entre las naciones como en el interior de las sociedades, los conflictos étnicos, la pobreza, el desempleo, la injusticia social, las migraciones masivas... que exigen un desarrollo concebido a escala mundial, donde la prosperidad de las sociedades esté fundada sobre los recursos humanos y el desarrollo de las capacidades de cada uno, sin distinción de ninguna clase. Por último, en consecuencia, la dignidad humana exige también el ejercicio para todos del derecho a una educación de calidad que favorezca el conocimiento y la comprensión mutua de los pueblos, la libre circulación de las ideas y el acceso de todos a los progresos de la ciencia y la tecnología.

\subsection{Los derechos humanos y la convivencia social}

$\mathrm{Si}$ convivir es, principalmente, vivir en comunidad estableciendo pautas y normas que favorezcan la ayuda, seguridad, colaboración y cooperación necesarias para, en primer lugar, satisfacer las necesidades humanas básicas a través del trabajo y el reparto equitativo de bienes $\mathrm{y}$, en segundo término, resolver eficazmente los conflictos de relaciones que se producen en el seno de esa comunidad. Las necesidades humanas y su satisfacción están en la base de ese conjunto de pautas y de normas, puesto que de la dialéctica entre las necesidades sentidas y la puesta en práctica de nuestras capacidades se construye el mundo de los valores. Valores que son socialmente aceptables cuando surgen de la generalización social de determinados grupos de necesidades, convirtiéndose, por consiguiente, en preferencias sociales compartidas por más de un grupo o sociedad. Según esta teoría, los derechos humanos forman el conjunto de normas, producto de esa dialéctica, con más consenso en la historia de la humanidad. Reconocer y asimilar aquellos valores morales que pueden entenderse como universalmente deseables es uno de los objetivos de la educación. Por otra parte, los derechos humanos, constituyen en el mundo relativista de los valores morales, el mínimum de una ética del consenso, garantizados por su universalidad (se imponen a todos los seres humanos los mismos derechos y las mismas obligaciones), por el principio de igualdad y por su doble naturaleza (emanan de la condición misma del ser humano y evolucionan como normas gracias a un proceso de construcción colectiva e histórica inacabado).

Es indudable que los derechos humanos (como jerarquización de valores) y su esperanzadora puesta en práctica en todas las políticas mundiales tiene una relación directa con la satisfacción de las necesidades fundamentales de las personas 
y de los grupos e indica el nivel de justicia social alcanzado, tanto en el interior de un país como en la esfera internacional. También el grado de respeto o inculcación de estos derechos revela el nivel de violencia estructural existente. Es evidente la relación estrecha entre necesidades, valores y derechos humanos. Como dice Galtung, la producción ha sido organizada de mala forma: "al nivel fundamental -suficiente comida, vestido y techo, un nivel razonable de salud, comunidad y educación- estas cinco necesidades habrían podido satisfacerse para todos. El fracaso de no satisfacerlas es evitable, lo que quiere decir que hay violencia presente" 51 . Mientras haya manifestaciones de violencias estructurales - hambre, marginación, racismo, desempleo, explotación, deuda externa, desequilibrios estructurales entre Norte/Sur, refugiados, deterioro de la naturaleza... - no puede ni haber paz ni darse las condiciones precisas para "bien convivir" ni entre los individuos de una sociedad entre sí, ni entre las naciones mismas. Es importante que los estudiantes consideren con detalle los valores, la dinámica y los resultados que acompañan el uso de la violencia directa, así como de la violencia estructural inmersa en el seno de nuestras sociedades. Y sobre todo, comprender y tomar conciencia de que la violencia no es la única, ni la más eficaz, de las maneras de afrontar los conflictos, a pesar de que esté presente como tal en nuestra sociedad y sea continuo eslogan en los medios de comunicación.

Que entre los valores éticos y la educación existe una recíproca relación de compromiso es una afirmación que nadie -en la actualidad- pone en tela de juicio. Junto al carácter normativo de la educación transcurren dimensiones múltiples de percibir, comprender y construir el mundo que imposibilitan que ésta se ubique al margen de la dimensión ética. Los valores pertenecen no sólo al mundo de lo real, sino también representan la utopía y la esperanza, el mundo de las aspiraciones y de los ideales.

Si la educación tiene como finalidad última el desarrollo integral de la persona, no puede negarse el valor de los derechos humanos y su implicación directa en su propia concepción. Esto por varias razones: en primer lugar, porque los derechos humanos conforman esa "ética del consenso" que rige la convivencia entre las personas de la misma

51. Galtung, Johan. Peace by Peaceful Jeans. Op. Cit. p. 9. o de diferente cultura; por esta razón deben ser en sí mismos contenidos tensionales propios de la enseñanza ya que son elementos básicos de los conocimientos del aprendizaje social. En segundo lugar, porque constituyen los cimientos de una cultura democrática basada en tres valores éticos esenciales: la libertad, el diálogo o debate y la participación. En tercer lugar, porque los derechos humanos constituyen los principios de una concepción educativa que fundamenta y orienta el currículo y el quehacer docente. Por último, porque sirven de elementos integradores de una concepción amplia de educación para la paz y permiten la posibilidad no sólo de ser los conductores que nos aproximen a la problemática mundial a través de los llamados ejes transversales, sino también de orientar desde una nueva perspectiva los conocimientos que provienen del mundo de la ciencia y de la tecnología.

Este cuerpo de principios y valores que conforman los Derechos Humanos encierra una tensión entre el carácter universal de los mismos y el respeto por los particularismos, ya que su evolución a lo largo de los siglos obedece a un proceso de respuesta continua a los problemas con que se han enfrentado las sociedades. Los derechos humanos deben entenderse como los elementos básicos de una ciudadanía que exige una actitud de respeto hacia la dignidad de la persona en su dimensión individual y colectiva, pero también al reconocimiento del otro y su peculiar forma de entender el mundo y, sobre todo, como respuesta a la sociedad en continuo cambio. En este sentido, la educación para la paz no puede entenderse como aquella educación de la ciudadanía que persigue el mantenimiento del statu quo, sino, en primer lugar, contribuir a la formación de individuos sociales capaces de promover la plena vigencia de los derechos humanos en una sociedad democrática y, por otro lado, favorecer la superación de los obstáculos que se oponen a este fin.

Así pues, los derechos humanos son fundamento de la convivencia y que deben ser utilizados para educar moralmente sin olvidar que éstos no sólo tienen una dimensión ética, sino también filosófica, social y jurídica. Por otra parte, no podemos reducir esa educación moral a la exclusividad de los derechos humanos, porque la convivencia en cualquier nivel contiene dimensiones también éticas ausentes, o no del todo recogidas, en los derechos humanos. 


\subsection{La educación para la paz, los derechos humanos y la democracia}

Los derechos humanos no tienen sentido en un mundo que niega las posibilidades legítimas de todos los seres humanos a ser felices y alcanzar el bienestar que les permitan satisfacer las necesidades básicas y un nivel de vida digno. Los derechos humanos se proclamaron no sólo para normalizar las relaciones de las personas en el seno de cualquier sociedad, sino también como un conjunto de criterios de valor con los que medir el progreso y la orientación de la sociedad misma. Sin duda, para satisfacer más eficazmente las necesidades materiales y espirituales de las personas, las sociedades necesitan de la participación de todos sus miembros a través de una organización social que los favorezca. La democracia, un buen gobierno e instituciones transparentes y responsables en todos los sectores de la sociedad son indispensables para la consecución de cualquier modelo de desarrollo centrado en el ser humano. Desarrollo que, por otro lado, necesita promover la cohesión e integración social a través de sociedades estables, seguras y justas, basadas en el ejercicio y respeto de los derechos humanos así como en la participación de todas las personas, incluidos los grupos y las personas desfavorecidos y vulnerables. El acceso de todos a la educación, la información, la tecnología y los conocimientos especializados son medios indispensables para mejorar la comunicación y aumentar la participación de todos los ciudadanos en la vida civil y la mejor manera de lograr el respeto de los derechos civiles, políticos, económicos, sociales y culturales como escribió H. Gros Espiell:

La enseñanza y la educación, en su sentido más amplio e integral, constituyen la esencia de la promoción de los derechos humanos, base ineludible y condición necesaria, aunque no exclusiva ni bastante, para el logro del respeto y la vigencia integral de estos derechos.

La educación para la paz, los derechos humanos y la democracia es considerada en la actualidad la finalidad esencial del derecho a la educación. Educación que por otra parte cuenta con directivas y leyes en muchos países y con el respaldo y la acción unánime de la sociedad civil ${ }^{52}$.

52. Página UNAMhttps://revistas-colaboracion jurídicas unam. $\mathrm{mx}$. fecha 25 de abril del 2015

\subsection{Retos de la Educación en materia de derechos humanos y cultura de la paz}

El reto principal de la educación consiste en colaborar en la tarea de la humanidad de tratar de encaminarse hacia formas futuras de organización social y de relaciones con el entorno que sean justas y ecológicamente perdurables. La educación, entendida como un proceso global de concienciación y de reconstrucción cultural de la sociedad, tiene como misión primera informar sobre el conocimiento cada vez más profundo de los problemas globales de la población mundial y del estado del planeta, de su desarrollo y tendencias, de los resultados de las indagaciones sobre sus causas y de los obstáculos que dificultan su resolución positiva; así como reflexionar sobre cómo los agentes sociales podrían promover las transformaciones necesarias.

La 44를 reunión de la Conferencia Internacional de Educación, organizada en octubre de 1994 por la Oficina Internacional de Educación de la Unesco. En dicho Plan leemos: "En un periodo de transición y de transformación acelerada caracterizado por la expresión de la intolerancia, las manifestaciones de odio racial y étnico, el recrudecimiento del terrorismo en todas sus formas y manifestaciones, la discriminación, la guerra y la violencia hacia el "otro" y las disparidades cada vez mayores entre ricos y pobres, tanto en el plano internacional como en el nacional, las estrategias de acción deben apuntar a garantizar las libertades fundamentales, la paz, los derechos humanos y la democracia, y a fomentar al mismo tiempo el desarrollo económico y social sostenible y equitativo ya que se trata de componentes esenciales de la construcción de una cultura de paz. Esto exige la transformación de los estilos tradicionales de la acción educativa.

La educación cumple una función esencial, cada vez mayor, como motor que contribuye al conocimiento y sensibilización de los miembros de la sociedad sobre los problemas mundiales $\mathrm{y}$, sobre todo, como medio de posibilitar la participación de todos los ciudadanos del mundo en la solución de dichos problemas. La educación así entendida, no sólo en su función socializadora sino también transformadora, ha encontrado en la Educación para la Paz (EP) una de sus mejores expresiones. Hoy no se concibe otra definición y finalidad de la educación que no sea ésta. Recordemos como esta misma explicación fue reconocida por los participantes en la Conferencia Mundial sobre Educación para Todos, reunidos 
en 1990 en la ciudad tailandesa de Jomtien: "La educación puede contribuir a lograr un mundo más seguro, más sano, más próspero y ambientalmente más puro y favorecer al mismo tiempo el progreso social, económico y cultural, la tolerancia y la cooperación internacional". ${ }^{53}$

Teniendo en cuenta las consideraciones anteriores, podemos definir la Educación para la Paz como un proceso dirigido tanto a los individuos, como a la sociedad, para que actúen conforme a los principios contenidos en la Declaración Universal de Derechos Humanos y todo el corpus jurídico internacional que los desarrolla, en favor del desarrollo sostenido de los pueblos, la protección y conservación del medio ambiente, la aspiración y acción en pro del desarme, el fortalecimiento de la convivencia social y la solución no violenta de los conflictos. Esta definición está sostenida por una concepción positiva de la paz como la situación caracterizada por "un nivel reducido de violencia y un nivel elevado de justicia" ${ }^{54}$, entendida esta última como la satisfacción de las necesidades humanas básicas a través de un desarrollo ambientalmente sostenido.

La educación para la paz se caracteriza por ser un proceso dinámico y permanente que pretende crear las bases de una nueva cultura: La cultura de la paz como expresión de las prácticas surgidas de aprender a pensar y actuar de otra manera, permitiendo un desarrollo equilibrado y armónico de las personas y las sociedades consigo mismas, con los demás y con el entorno natural. Esa conciencia holística permite, por tanto, una conciencia cósmica y ecológica que, en el plano educativo, se traduce en la superación del viejo paradigma fundado en la fragmentación de la ciencia y del conocimiento, de modo que la educación era considerada principalmente como la enseñanza dirigida al desarrollo de la capacidad intelectual y sensible. Esta visión global de la paz y de la cultura entiende que la función educativa, en su nueva interpretación, no es único objetivo de la escuela sino que su responsabilidad recae en todos los elementos del entramado social y demuestra

53. UNESCO. Informe del director general sobre los trabajos del Congreso Internacional sobre Educación para los Derechos Humanos y la Democracia. Montreal. 1993. Consultado en: http://unesdoc.unesco.org/images/0009/000954/095433so. pdf fecha 25 de abril del 2015

54. CURLE, Adam. Education for Liberation, J.Wiley, Nueva York. 1973 que experiencia en la vida de las personas constituye una oportunidad para aprender.

E el Congreso Internacional sobre Educación en Derechos Humanos y en Democracia, celebrado en Montreal en marzo de 1993, se proclamó ${ }^{55}$ que los valores democráticos son un requisito para el ejercicio efectivo de los derechos humanos. La declaración surgida de este congreso establece: a) La necesidad de la enseñanza y de la educación en los valores democráticos como requisito para el ejercicio de los derechos humanos; b) Dicha educación es en sí un derecho fundamental y condición esencial para el pleno desarrollo de la justicia social, de la paz y del desarrollo; c) La educación en derechos humanos y en democracia es un instrumento valioso de protección de esos derechos y de prevención contra cualquier tipo de abusos; d) La finalidad de dicha educación debe alcanzarse a través de un proceso dinámico basado en la participación.

Inspirado notablemente en la siempre referida Recomendación de UNESCO de 1974, así como en las recomendaciones formuladas en el Congreso de Viena de 1978 (sobre la enseñanza de los Derechos Humanos), en el Congreso de Malta de 1987 (sobre la enseñanza, la información y la documentación en materia de derechos humanos) y en el Foro Internacional "La educación para la democracia" de Túnez de 1992, el Plan de Acción Mundial para la Educación en los Derechos Humanos y en la democracia de Montreal, remoza y contextualiza las directrices y fundamentos de una educación que preconiza el aprendizaje de la tolerancia, la aceptación del "otro", de la solidaridad y de la ciudadanía fundada en la participación. En el momento actual un enfoque moderno de los problemas relativos a la educación para la paz, los derechos humanos y la democracia está contenido en el Plan de Acción Integrado surgido de la Conferencia Internacional de Educación de 1994. Este documento señala las finalidades de dicha educación, las estrategias de acción y las políticas y orientaciones en los planos institucional, nacional e internacional. Además representa un nuevo intento de garantizar -a través de la educación- las libertades fundamentales, la paz, los derechos humanos y la democracia, y de fomentar al mismo tiempo el desarrollo económico y social sostenible

55. Pagina de internet https:77www.latarea.com.mx.separa. fecha 25 de abril del 2015 
y equitativo, ya que se trata de componentes esenciales de la construcción de una cultura de paz. Dicho plan, después de justificar en su introducción la necesidad de este tipo de educación, establece las siguientes finalidades:

-La educación ha de fomentar la capacidad de apreciar el valor de la libertad y las aptitudes que permitan responder a sus retos. Ello supone que se prepare a los ciudadanos para que sepan manejar situaciones difíciles e inciertas, prepararlos para la autonomía y la responsabilidad individuales. Esta última ha de estar ligada al reconocimiento del valor del compromiso cívico, de la asociación con los demás para resolver los problemas y trabajar por una comunidad justa, pacífica y democrática.

-La educación debe desarrollar la capacidad de reconocer y aceptar los valores que existen en la diversidad de los individuos, los sexos, los pueblos y las culturas, y desarrollar la capacidad de comunicar, compartir y cooperar con los demás. Los ciudadanos de una sociedad pluralista y de un mundo multicultural deben ser capaces de admitir que su interpretación de las situaciones y de los problemas se desprende de su propia vida, de la historia de su sociedad y de sus tradiciones culturales y que, por consiguiente, no hay un solo grupo que tenga la única respuesta a los problemas, y puede haber más de una solución para cada problema. Por tanto, las personas deberían comprenderse y respetarse mutuamente y negociar en pie de igualdad con miras a buscar un terreno común. Así, la educación deberá fortalecer la identidad personal y favorecer la convergencia de ideas y soluciones que refuercen la paz, la amistad y la fraternidad entre los individuos y los pueblos.

- La educación debe desarrollar la capacidad de resolver los conflictos con métodos no violentos. Por consiguiente, debe promover también el desarrollo de la paz interior en la mente de los estudiantes para que puedan asentar con mayor firmeza las dotes de tolerancia, solidaridad, voluntad de compartir y atención hacia los demás.

- La educación ha de cultivar en el ciudadano la capacidad de hacer elecciones con conocimiento, basando sus juicios y sus actos no sólo en el análisis de las situaciones actuales, sino también en la visión de un futuro al que aspira.
- La educación debe enseñar a los ciudadanos a respetar el patrimonio cultural, a proteger el medio ambiente y a adoptar métodos de producción y pautas de consumo que conduzcan al desarrollo sostenible. También es indispensable la armonía entre los valores individuales y los colectivos y entre las necesidades básicas inmediatas y los intereses a largo plazo.

- La educación ha de nutrir sentimientos de solidaridad y equidad en los planos nacional e internacional en la perspectiva de un desarrollo equilibrado y a largo plazo.

- Preparación para la no-violencia: Preparar a nuestros jóvenes en el pensamiento y prácticas de la no-violencia es uno de los objetivos básicos de una educación basada en la búsqueda de nuevas formas de resolver los conflictos y de construir una paz basada en la justicia. Y esto es obvio pues ni los contenidos, los objetivos y las formas de educar para la paz pueden ser contrarias a la finalidad última que este tipo de educación persigue.

- Responsabilidad de los ciudadanos del mundo: En todos los procesos de interacción social se precisa un mínimo de responsabilidad. La responsabilidad no consiste sólo en cumplir las obligaciones y deberes, sino que además supone captar los rasgos morales de esta relación, actuar conforme a ellos, situarse en el mundo, conocer sus problemas y tomar conciencia de la necesidad de cambio. Es decir, adoptar un comportamiento ético ante las cosas que pasan ante nuestras miradas, en nuestra proximidad más cercana, como individuos y seres sociales, y, también, en esa aldea global en la que todos vivimos. Ubicarse en el mundo significa dar respuesta a sus interrogantes, una respuesta que debe comenzar por ser individual, pero que también ha de ser compartida colectivamente. La responsabilidad es un rasgo esencial de la experiencia moral de los individuos y de la comunidad, del desarrollo de un aprendizaje que permite la consolidación autónoma de una actitud ética frente al mundo y de una conciencia planetaria. Sin duda que los problemas con los que se enfrentan los ciudadanos de todos los países no pueden resolverse sin una construcción ética basada en la afirmación de espacios cada vez mayores de autonomía donde se desacralice la autoridad y, por otro lado, se intente humanizarla.

- Igualdad de actitudes: La educación para la paz es una forma particular de educación en 
valores que persigue el desarrollo de actitudes iguales en todos los jóvenes del mundo, de ahí su vocación internacional, para que ante valores antitéticos a la cultura de la paz como la obediencia ciega, el conformismo y consumismo, la indiferencia e insolidaridad, la intolerancia o la discriminación se cuestionen sus consecuencias y actúen guiados por la justicia, la tolerancia y la solidaridad. Soluciones estables a los problemas de rápido crecimiento demográfico, de pobreza extrema, de desintegración social y de desigualdad entre hombres y mujeres en todo el mundo, dependen, como fue reconocido en la Cumbre Mundial para el Desarrollo Social, de la formación de los jóvenes en los conocimientos, capacidades y actitudes necesarias para instaurar una solidaridad internacional, favoreciendo el pluralismo, la tolerancia, la igualdad entre sexos y el interés por el otro. Actitudes que comprometen a todos y a todas en un sentimiento de comunidad y de cooperación mundial. De aquí la necesidad de que los temas controvertidos como la violencia, la desigualdad, los conflictos armados, la discriminación y tantos otros reciban una atención especial en las instituciones educativas con el fin de adecuar el currículo a las exigencias de nuestro tiempo.

- Investigación crítica de alternativas: La educación para la paz es crítica con la realidad, pero también creativa porque la creatividad está en la propia dimensión humana. Enfrentarse a los problemas que genera la insolidaridad no sólo es un gran sueño sino una emergencia. La educación no puede quedarse con los brazos cruzados ante tales atrocidades, sino que tiene que, tocando tierra, imaginar nuevos futuros probables, posibles y deseables. La educación tiene como misión hacer que los jóvenes examinen los obstáculos que a menudo nos impiden experimentar un progreso hacia la paz; familiarizarles con destrezas específicas que venzan esas dificultades y brindarles modelos de personas y de grupos cuyas acciones se encaminan en pro de una paz realizable.

\subsection{Contenidos que debe incluirse en los currículos en materia de derechos humanos y cultura de la paz}

a). Aspectos éticos y cívicos: Conductas y actitudes basadas en el reconocimiento de la igualdad y en la necesidad de la interdependencia de las naciones y de los pueblos; eliminación de todas las formas de discriminación; ejercicio y respeto de los derechos humanos; conocimiento del funcionamiento y obra de las instituciones nacionales e internacionales para resolver los problemas nacionales e internacionales; análisis crítico de los factores que impiden la paz.

b). Aspectos culturales: Estudio de diferentes culturas.

c). Estudio de los principales problemas de la humanidad: Igualdad de derechos de los pueblos; mantenimiento de la paz, derechos humanos, el desarrollo económico y social y su relación con la justicia; problemas de deterioro medioambiental, etc.

d). Una formación que proporcione los suficientes conocimientos, habilidades, destrezas, actitudes y valores para vivir y mejorar la democracia en la familia, la escuela y la sociedad.

e). Unas prácticas que permitan experimentar y sentir en la propia piel los principios de participación, solidaridad, justicia y libertad.

f). Una atmósfera (familiar, escolar y social) que facilite esa vivencia.

g). La posibilidad de deliberar y decidir que estimule el pensamiento crítico sobre la democracia vivida o percibida en la familia, la escuela o la sociedad.

\subsection{El papel del docente}

Estos temas que hoy en día se analizan de forma transversal, exigen desde la educación para la paz, los derechos humanos y la democracia - un modelo de perfeccionamiento del profesorado que se sitúa en un nuevo paradigma formativo orientado no tanto a la adquisición de técnicas y métodos como a modificar la función y el papel del profesorado tanto en el seno de la sociedad (agente social) como en el interior de la escuela (agente docente). Por consiguiente, la formación recibida debe prepararle para desempeñar esta función de la manera más adecuada Además, como tutor de un grupo de alumnos y como miembro de una comunidad educativa, debe fomentar las siguientes capacidades:

Capacidad de crear un clima escolar que favorezca el diálogo, el intercambio de ideas y la construcción de nuevos conocimientos. 
- Capacidad de crear situaciones en la que el alumnado pueda vivir experiencias conflictivas desde el punto de vista moral que le obliguen a actuar moralmente y reciclar sus dimensiones en este ámbito.

- Capacidad de escuchar, de aconsejar y de ayudar en la formación y en el desarrollo integral de su alumnado, motivándoles y estando atento a sus intereses e inquietudes.

- Capacidad para construir un modelo teórico propio y adaptado a la situación educativa concreta, adaptando y recreando, cuando sea necesario, todas y cada una de las actividades propuestas.

- Capacidad de animar a los grupos y de analizar su funcionamiento, de comprender el sentido y la dinámica de las situaciones que se plantean en las aulas.

- Capacidad de trabajo sobre la propia persona y de interrogarse sobre uno mismo dentro de su práctica docente que le permita poseer un autoconcepto ajustado y positivo que le facilite el ejercicio de su función.

- Capacidad para dirigir discusiones morales que le exige tener la competencia para afrontar y manejar situaciones desde un punto de vista moral y Ético

\section{CONCLUSIONES}

Primera. La implementación del nuevo sistema de justica ha exigido un cambio radical tanto en los ordenamientos legales, así como en la infraestructura, los métodos y los procesos institucionales. Aunado a lo anterior el operario del derecho sea cual sea su posición, se enfrenta ante el reto de cambiar su dinámica en el ejercicio profesional, requiere de una nueva capacitación para asumir los retos de este nuevo paradigma jurídico, deberá ser capaz de desarrollar competencia en comunicación verbal, oratoria, aunado a la integración en su ejercicio profesional de la cultura de la paz y el respecto a los derechos humanos.

Segunda. La propia UNESCO entiende que la alternativa a la cultura de la no violencia debe sustentarse en la negociación, el diálogo, la mediación, el empoderamiento, la empatía, y la capacidad de manejar nuestros propios conflictos, así como el respecto a los derechos humanos.

Tercera. El futuro exige más que nunca la construcción de la paz, a través de la ciencia, la cultura, la educación y la comunicación, debido a que el respeto al derecho humano a la paz inspirado en el ideal democrático de dignidad, igualdad y respeto de la persona es la vía más segura para luchar contra la exclusión, la discriminación, la intolerancia y la violencia que amenazan la cohesión de las sociedades y conducen a los conflictos armados.

Cuarta. La educación del ciudadano no puede ser responsabilidad exclusiva del sector educativo por lo que éste debe cooperar estrechamente, para cumplir eficazmente sus funciones, con los demás agentes de socialización, entre ellos: la familia, los medios de comunicación, el mundo del trabajo y las organizaciones no gubernamentales.

Quinta. Los principios rectores de la cultura de paz son fundamentales para instaurar un orden de justicia y bienestar, pero no bastan en sí mismos para lograr la paz social, pues hace falta compromiso $\mathrm{y}$ acciones concretas para darles vigencia.

Sexta. Los estados deben fortalecer la cultura de paz, promoviendo la plena vigencia de los derechos humanos, utilizando la educación como una herramienta transformadora, trabajando para superar las desigualdades y fortaleciendo las instituciones democráticas, es decir, tomando las medidas necesarias para asegurar una convivencia pacífica. Sin embargo, la promoción de la paz no es una tarea exclusiva de los estados, ya que la sociedad civil también está llamada a contribuir activamente en el reto de neutralizar la violencia, de modo que se promueva y se practique la paz a nivel comunitario, vecinal, escolar, etcétera.

Séptima. La cultura de paz guarda estrecha relación con los derechos humanos, pues al promover la comprensión, el respeto y la solidaridad, es posible lograr una convivencia más justa, libre y respetuosa de la dignidad humana. Claramente, se trata de una tarea compleja que requiere el compromiso de parte de las autoridades, pero que sobre todo necesita el apoyo de todas y todos para generar cambios estructurales que faciliten la convivencia en un contexto de plena vigencia de los derechos humanos. 


\section{BIBLIOGRAFÍA}

- AGUILERA, Rafael. "La Mediación. Un acer camiento real a la Justicia y a la Cultura de Paz." En Gorjón Gómez, Francisco Javier, et al. Mediación y Arbitraje. Leyes Comentadas y Concordadas del Estado de Nuevo León. Ed. Porrúa. México. 2009.

- AIELlO DE ALMEIDA, María Alba, Mediación: Formación y algunos aspectos claves. Ed. Porrúa. México. 2010.

- APUD. ROMERA, Raúl. Guerra, posguerra y paz. Pautas para el análisis y la intervención en contextos posbélicos o postacuerdo. Ed. Icaria. Barcelona. 2003.

- AZAR MANSUR, Cecilia. Mediación y Conciliación en México: Dos vías alternativas de solución de conflictos a considerar. Ed. Porrúa. México. 2003.

- BARBOSA RAMOS, Isaac. El Valor del Perdón. Ed. Selector. México. 2006.

- BOLIVAR, Antonio. La evaluación de valores y actitudes. Alauda-Anaya, Madrid. 1995.

- BJERSTEDT, Ake. La educación para la paz hoy y mañana: visiones breves de una encuesta internacional actual y algunas reflexiones sobre los objetivos de la Educación para la Paz. Informe presentado en la Conferencia del IPRA, Universidad de Sussex. 1986.

- BUXARRAIS, Ma Rosa. La formación del profesorado en educación en valores. Propuesta y materiales. Colección Aprender a ser, editorial Desclée De Brouwer, Bilbao. 1997.

- CAMPS, Victoria. Los valores de la educación, Anaya, Madrid. (1994)

- CURLE, Adam. Education for Liberation, J.Wiley, Nueva York. 1973

- DÍAZ, Luis. Nuevas configuraciones del Derecho a la Paz. Revista Misión Jurídica. Ed. Universidad Colegio Mayor de Cundinamarca. Bogotá. No. 9. 2015. pp. 259 - 277

- EllacuRIA, I. " Historización de los Derechos Humanos desde los pueblos oprimidos y las mayorías populares", en Revista ECA, año XLV, no 502 , agosto,
Universidad Centroamericana "José Simeón Cañas”, El Salvador, 1990, p.590.

- FISAS, VINCES. Cultura de paz y gestión de conflictos. Ed. Icaria/Antrazyt. UNESCO. Barcelona, 2001.

- GOTTHEIL, JULIO. "La mediación y salud del tejido social". En Gottheil, Julio y Schiffrin, Adriana (comp.) Mediación: una transformación de la cultura. Ed. Paídos. Buenos Aires 1996. p. 215-225

- GAlTuNG, Johan. Peace by Peaceful Jeans. Peace and Conflict, Development and Civilization. Ed. Prio, Oslo, 1996.

- Galtung, Johan. The True Worlds, The Free Press, New York, 1981.

- GALTUNG, Johan. "Cultural Violence", Journal of Peace Research 3, vol.27, 291315. 1990.

- HOWE, L. W. y HOWE, M. Cómo personalizar la educación. Perspectivas de la clarificación de valores, Santillana, Madrid, 1997.

- LEDERACH, John Paul. Construyendo la paz. Reconciliación sostenible en sociedades diversas. Ed. Bakeaz, Bilbao, 1998.

- MAGENDZO, Abraham. "Perfeccionamiento docente en educación y derechos humanos: reflexiones a partir dela experiencia" en Educación en Derechos Humanos: Apuntes para una nueva práctica, Corporación Nacional de Reparación y reconciliación, Santiago de Chile, 1994.

- MAYOR ZARAGOZA, Federico. "Educación para la Paz" en Educación XXI: Revista de la Facultad de Educación. Ed. UNED. Madrid, No. 6. 2003.

- MAYOR ZARAGOZA, Federico. El derecho humano a la paz, UNESCO, París. (SHS-97/ WS/6). 1997.

- PÉREZ,José Benito,ZARAGOZA,JoséyBARBA ALVAREZ, Rogelio. "La interdisciplinariedad y multidisciplinariedad como modelos a seguir en la enseñanza del Derecho: La experiencia de los Métodos Alternos de Solución de Controversias" en Letras 
Jurídicas: revista electrónica de Derecho. Universidad de Guadalajara. Primavera del 2009. N. 8 http://letrasjuridicas. cuci.udg.mx /numeros/articulos 8 / La $\% 20$ interdisciplinariedad $\% 20 y \% 20$ multidisciplinariedad.pdf $V$. Página de la Revista: http://letrasjuridicas.cuci.udg.mx/

- REARDON, BETTY. Militarización, security and peace education. United ministres in education, USA, 1982.

- TUVILLA, J. Derechos Humanos: propuesta de educación para la paz basada en los derechos Humanos y del Niño, CEJA, Sevilla, 1990.

- ZABALA, A. "La evaluación de los valores en el currículum" en Educación En Valores $Y$ Temas Transversales Del Curriculum, Centro del profesorado, Delegación Provincial de Educación y Ciencia, Almería, 1997. 